\title{
A CHARACTERIZATION IN THE SPACE OF CONVOLUTION OPERATORS
}

\author{
B. R. NAGARAJ
}

ABSTRACT. We give a characterization of $C^{\infty}$ elements in the space of convolution operators $\theta_{c}^{\prime}$, which belong to the Schwartz space $\mathscr{S}$.

In the space of convolution operators $\theta_{c}^{\prime}$, also referred to as the space of distributions which are rapidly decreasing at $\infty$, there are $C^{\infty}$ elements which are not rapidly decreasing in the sense of the Schwartz space $\mathscr{S}$. For a preliminary discussion of the spaces $\theta_{c}^{\prime}$ and its image $\theta_{M}$ under the Fourier transform, see Treves [5, pp. 314-321], or Schwartz [4, Chapter VII, §8].

The purpose of this note is to give a necessary and sufficient condition for $C^{\infty}$ elements in $\theta_{c}^{\prime}$ to belong to $\mathscr{S}$. Notation used here but not defined is standard; see Hörmander [1, 2].

Let $\rho(\xi) \in \mathscr{S}^{m}$ be any "constant coefficient" symbol, that is, $\rho(\xi) \in C^{\infty}\left(\mathbf{R}^{n}\right)$ and satisfies for each multi-index $\alpha$ the estimate

$$
\left|D_{\xi}^{\alpha} \rho(\xi)\right| \leqslant c_{\alpha}(1+|\xi|)^{m-|\alpha|}, \quad \xi \in \mathbf{R}^{n},
$$

where $c_{\alpha}$ are constants. Let $\rho(D)$ be the pseudo-differential operator corresponding to the symbol $\rho(\xi)$. Then clearly, for $v \in \mathscr{E}^{\prime}$, one has

$$
\widehat{\rho(D) v}(\xi)=\rho(\xi) \hat{v}(\xi) \in \theta_{M} .
$$

Thus $\rho(D)$ may be considered as a map $\rho(D): \mathscr{E}^{\prime} \rightarrow \theta_{c}^{\prime}$ and is sometimes referred to as a Friedrichs operator. With this notation our main result becomes the following

THEOREM. Let $f \in \theta_{c}^{\prime} \cap C^{\infty}\left(\mathbf{R}^{n}\right)$. Then $f \in \mathscr{S}$ if and only if there exists a Friedrichs operator $\rho(D)$ such that $f=\rho(D) v$ for some distribution $v$ with compact support.

REMARK. A weaker form of the Theorem, namely that if $\chi(\xi)$ is a $C^{\infty}$ function on $\mathbf{R}^{n}$, positively homogeneous of degree 0 for $|\xi|>1$ and if $v \in \mathscr{E}^{\prime}$ is such that $\chi(D) v$ is $C^{\infty}$, then $|\chi(\xi) \hat{v}(\xi)|=O\left(|\xi|^{-N}\right)$ for all positive integers $N$, has been mentioned in Nirenberg [3, p. 42].

The proof of the Theorem is based on the following

Lemma. $\left\{\rho(D) v: v \in \mathscr{E}^{\prime}\right\} \cap C^{\infty}\left(\mathbf{R}^{n}\right) \subset \mathscr{S}$.

Received by the editors January $16,1984$.

1980 Mathematics Subject Classification. Primary 46F10, 35 S 99.

(C)1985 American Mathematical Society $0002-9939 / 85 \$ 1.00+\$ .25$ per page 
Proof. Suppose $\rho(D) v \in C^{\infty}\left(\mathbf{R}^{n}\right)$ for some $v \in \mathscr{E}^{\prime}, v \neq 0$. Then we may write (see [2, Theorem 2.13, p. 149])

$$
\rho(D)=\rho_{1}+\rho_{2}
$$

where $\rho_{1}$ is properly supported, that is, maps $C_{0}^{\infty}$ into itself and $\mathscr{E}^{\prime}$ into itself; $\rho_{2}$ has a $C^{\infty}$ kernel and defines a map on $\mathscr{E}^{\prime} \rightarrow C^{\infty}\left(\mathbf{R}^{n}\right)$. This decomposition is defined by a decomposition of the Schwartz kernel $K_{\rho}$ associated with $\rho(D)$, in the form

$$
K_{\rho_{1}}=\theta K_{\rho} \text { and } K_{\rho_{2}}=(1-\theta) K_{\rho}
$$

where $\theta \in C^{\infty}\left(\mathbf{R}^{n} \times \mathbf{R}^{n}\right)$ which takes values in [0,1], equals 1 in a neighbourhood of the diagonal of $\mathbf{R}^{n} \times \mathbf{R}^{n}$ and is properly supported.

The expression for the kernel $K_{\rho}$ is given by

(4) $K_{\rho}(x, y)=\frac{(2 \pi)^{-n}}{(x-y)^{\alpha}} \int_{\mathbf{R}^{n}} e^{i(x-y) \cdot \xi}\left(-D_{\xi}\right)^{\alpha} \rho(\xi) d \xi, \quad x, y \in \mathbf{R}^{n}, x \neq y$,

where the multi-index $\alpha$ is arbitrary but chosen so that the integral converges absolutely. Now by (2), we have

$$
\rho(D) v=\rho_{1}(v)+\rho_{2}(v) \Rightarrow \rho_{1}(v) \in C_{0}^{\infty}\left(\mathbf{R}^{n}\right) .
$$

Our aim now is to prove that $\rho_{2}(v) \in \mathscr{S}$. To this end, we shall first establish the form of the $C^{\infty}$ function $\rho_{2}(v)$. If we choose $f \in C_{0}^{\infty}\left(\mathbf{R}^{n}\right)$ such that $f=1$ in a neighbourhood of $\operatorname{supp}(v)$, then (see [2, Definition 2.11, p. 148])

$$
\rho_{2}(v)(x)=\rho_{2}(f v)(x)=(2 \pi)^{-n} \int_{\mathbf{R}^{n}} e^{i x \cdot \xi} p_{f}(x, \xi) \hat{v}(\xi) d \xi
$$

where $p_{f}$ stands for a symbol of $\rho_{2}$ in $\mathscr{S}^{-\infty}$ and is given by the expression

$$
e^{i x \cdot \xi} p_{f}(x, \xi)=\rho_{2}\left(f e^{i\langle, \xi\rangle}\right)(x)
$$

where $e^{i\langle, \xi\rangle}$ denotes for each $\xi$ the function $e^{i x \cdot \xi}$. Now expressing the action of $\rho_{2}$ in terms of its kernel, we have from (3) and (4)

$$
\rho_{2}\left(f e^{i\langle, \xi\rangle}\right)(x)=(2 \pi)^{-n} \int_{\mathbf{R}^{n}}\left[\frac{(1-\theta)}{(x-y)^{\alpha}} \int_{\mathbf{R}^{n}} e^{i(x-y) \cdot t}\left(-D_{t}\right)^{\alpha} \rho(t) d t\right] f(y) e^{i y \cdot \xi} d y .
$$

Noting that $\theta(x, y) f(y)$ has compact support since $\theta$ is properly supported and integrating by parts with respect to $y$, we obtain, for arbitrary multi-indices $\beta_{1}, \beta_{2}$

$$
\sup _{(x, \xi) \in \mathbf{R}^{n} \times \mathbf{R}^{n}}\left|\xi^{\beta_{1}} D_{x}^{\beta_{2}} \rho_{2}\left(f e^{i<, \xi\rangle}\right)(x)\right|<\infty
$$

when $\alpha$ is chosen so that $|\alpha|>\left(m+n+\left|\beta_{1}\right|+\left|\beta_{2}\right|\right)$. Now from (6) and (7) we have

$$
\rho_{2}(v)(x)=(2 \pi)^{-n} \int_{\mathbf{R}^{n}} \hat{v}(\xi) \rho_{2}\left(f e^{i\langle, \xi\rangle}\right)(x) d \xi
$$

where we note that $\hat{v}$ has polynomial growth, say of order $N$, and the integral is absolutely convergent because of (9). Differentiating under the integral sign in (10), it is clear that for arbitrary multi-indices $\mu, \nu$ we have

$$
\sup _{x \in \mathbf{R}^{n}}\left|x^{\mu} D_{x}^{\nu} \rho_{2}(v)(x)\right|<\infty
$$


when the multi-index $\alpha$ appearing in (8) is chosen large enough, that is,

$$
|\alpha|>\max \{|\mu|, m+2 n+N+1+|\nu|\} \text {. }
$$

Thus, $\rho_{2}(v) \in \mathscr{S}$. Hence by (5) $\rho(D) v \in \mathscr{S}$ and the proof of the Lemma is complete.

Proof of The TheOREM. $f \in \mathscr{S} \Leftrightarrow \hat{f}(\xi) \in \mathscr{S} \Leftrightarrow \hat{f}(\xi) \in \mathscr{S}^{m} \forall$ real $m$. Also we have $f=\hat{f}(D) \delta$, where $\delta$ is the Dirac measure on $\mathbf{R}^{n}$. The rest of the proof follows from the Lemma.

\section{REFERENCES}

1. L. Hörmander, Linear partial differential operators, Grundleheren Math. Wiss., Vol. 116, SpringerVerlag, Berlin and New York, 1963.

2. . Pseudo-differential operators and hypoelliptic equations, Singular Integrals, Proc. Sympos. Pure Math., vol. 10, Amer. Math. Soc., Providence, R. I., 1967.

3. L. Nirenberg, Lectures on linear partial differential equations, CBMS Regional Conf. Ser. in Math., no. 17, Amer. Math. Soc., Providence, R. I., 1972.

4. L. Schwartz, Théorie des distributions, 2nd ed., Hermann, Paris, 1966.

5. F. Treves, Topological vector spaces, distributions and kernels, Academic Press, New York, 1967.

Tata Institute of Fundamental Research, Bangalore Centre, Post Box 1234, Bangalore 560012, INDIA 